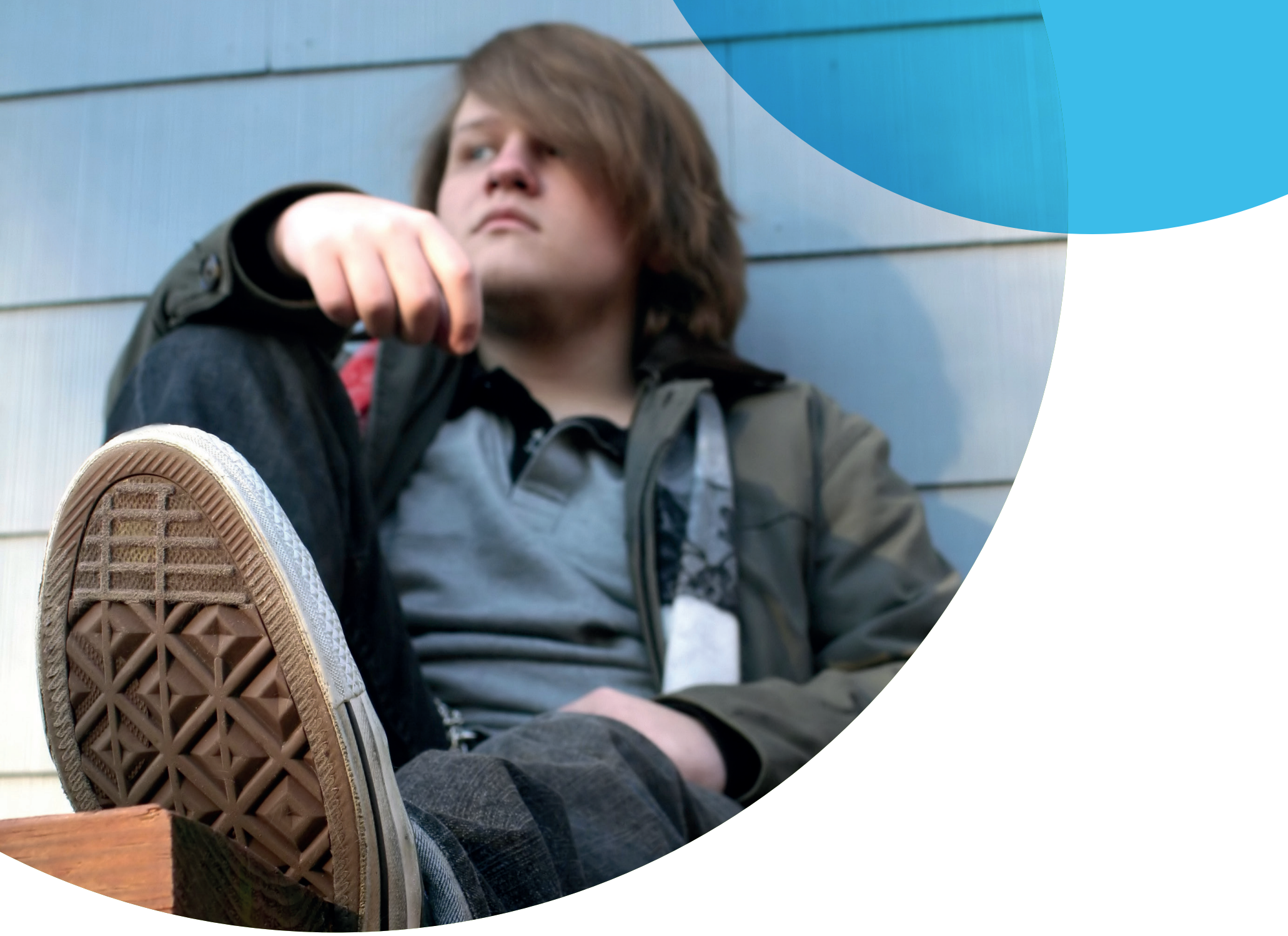

\title{
Children with ASD at heightened risk for maltreatment
}

\section{By Dr. Jessica K. Edwards}

A study conducted by researchers at the University of South Carolina is the first to provide empirical evidence from population-level data that maltreatment is elevated in children with autism spectrum disorder (ASD) relative to the general population. The researchers correlated data obtained from the Department of Social Services (DSS) and the Autism and Developmental Disabilities Monitoring (ADDM) network and found that children with ASD, intellectual disability (ID) or both had a significantly higher likelihood ( 2.5 times) of reported and substantiated maltreatment compared to typically developing controls. Regarding the characteristics of maltreatment, children in all learning difficulty groups experienced physical neglect. Children with ASD and co-morbid ID, or ID alone were more likely to experience all forms of abuse compared to those with ASD alone and typically developing controls, whereas those with ASD alone were more likely to experience physical abuse.

Finally, the researchers found that maltreated children with ASD were more likely to exhibit aggression, hyperactivity and tantrums than nonmaltreated children. Going forward, the researchers explain that empirically supported assessment and intervention approaches are urgently needed to identify maltreatment in children with ASD and ID.

\section{Referring to:}

McDonnell, C.G., Boan, A.D., Bradley, C.C., Seay, K.D., Charles, J.M. and Carpenter, L.A. (2018), Child maltreatment in autism spectrum disorder. J. Child Psychol. Psychiatr. doi: 10.1111/jcpp.12993. 